\title{
Eccentricity Compensator for Log-Polar Sensor
}

\author{
Sota Shimizu, Member, IEEE, Joel W. Burdick, Member, IEEE,
}

\begin{abstract}
This paper aims at acquiring robust rotation, scale, and translation-invariant feature from a space-variant image by a fovea sensor. A proposed model of eccentricity compensator corrects deformation that occurs in a log-polar image when the fovea sensor is not centered at a target, that is, when eccentricity exists. An image simulator in discrete space remaps a compensated log-polar image using this model. This paper proposes Unreliable Feature Omission (UFO) that reduces local high frequency noise in the space-variant image using Discrete Wavelet Transform. It discards coefficients when they are regarded as unreliable based on digitized errors of the input image. The first simulation mainly tests geometric performance of the compensator, in case without noise. This result shows the compensator performs well and its root mean square error (RMSE) changes only by up to $2.54[\%]$ in condition of eccentricity within $34.08\left[^{\circ}\right]$. The second simulation applies UFO to the log-polar image remapped by the compensator, taking its space-variant resolution into account. The result draws a conclusion that UFO performs better in case with more white Gaussian noise (WGN), even if the resolution of the compensated log-polar image is not isotropic.
\end{abstract}

\section{INTRODUCTION}

$\mathrm{T}$ HE log-polar mapping, inspired by analytic formulation of biological study of the primate visual system [1], is a method of space-variant imaging that can reduce image data size drastically. Some computational models mapping the log-polar image from a uniform-resolution image have been reported [2][3]. In order to obtain the log-polar image, 2 types of actual fovea sensors have been proposed and developed. One is by a special CCD or CMOS log-polar chip, where united size of each photosensitive element increases as going to periphery [5]-[7]. The other is by a special fovea lens, such as a Wide-Angle Foveated (WAF) lens, where a projected image is distorted geometrically [8]-[10].

One of the most remarkable advantages of the log-polar image is to give us rotation and scale-invariant (RS-invariant) property [5][10], because its image resolution distributes isotropically and changes logarithmically from the image center towards the periphery. Assume a situation where a view direction (that is, the optical axis) of the fovea sensor is perpendicular to a planar target image (that is, without shear).

Manuscript received September 6, 2006. This work was supported in part by Wind \& Biomass Energy R\&D and Information Center, and. a grant from Japan Society for the Promotion of Science.

Sota Shimizu is with Division of Biology, California Institute of Technology , Pasadena, CA, 91125, USA (phone: +1-626-395-2359; e-mail: sato@caltech.edu).

J.W.Burdick is with Division of Engineering and Applied Science, California Institute of Technology, Pasadena, 91125, USA (e-mail: jwb@robotics.caltech.edu).
If the fovea sensor is directed towards the image center of the target image accurately, we can obtain the RS-invariant feature from the image that is located at different distances and rotated about the optical axis. But if the fovea sensor is not centered at the image center correctly, a positional error between the optical axis and the image center (that is, so called, positional eccentricity) causes geometric deformation in the log-polar image and reduces the RS-invariant property (see Fig. 1(d) and (f), and also refer to [5]). We need to differentiate the deformation and image distortion. It should be noted that the latter occurs inherently due to space-variant sampling by the fovea sensor.

This paper discusses a method for acquiring rotation, scale, and translation-invariant (RST-invariant) feature, robust to the eccentricity and noise, from the log-polar image by the fovea sensor. The following 2 points are focused mainly.

1) Remapping a new log-polar image from the original log-polar image by compensating the eccentricity in order to increase robustness of geometric RS-invariant property.

2) Removing unreliable Wavelet Transform coefficients of the remapped image by taking space-variant resolution into account in order to improve image quality.

In terms of the first issue, we can see relevant ideas in some active visual tracking researches using the log-polar image (e.g. image warping technique [11] and a concept of virtual retina [12]). They are characterized as follows:

(a) Cartesian coordinates have been used for computing image motion (that occurs due to active camera motion).

(b) A remapped log-polar image (or displacement in the image) has been computed from the original image using the image motion.

In Cartesian space, translation caused by the positional eccentricity can be compensated easily. This property seems to have led to applying the affine model to compute the image motion [11][13]. In addition, the log-polar space is compatible with representing rotation and scaling [13]. Each of these ideas has performed its following correlation process well to increase stabilization of visual tracking in the central field of view (so-called, the fovea). However, it seems that the above researches have not discussed deeply enough with respect to quality of the remapped image (or the computed displacement), although it is space-variant. This problem motivates the second issue that pays attention to resolution of the log-polar image remapped from the original one, in order to estimate reliability of image components. The resolution of the log-polar image is space-variant inherently. In addition 
to that, that of the remapped one is anisotropic from the image center (see Fig. 1(c) and note the eccentricity gives space-variant resolution that is not isotropic, even if the deformation is corrected). Discrete Wavelet Transform (DWT) is well known as multi-resolution analysis [15] and it has been applied to foveation of the image [16]. The authors think this structure is suitable for representing the anisotropic log-polar image as mentioned above.

This paper is organized in the following way. Chapter II proposes a model of eccentricity compensator that corrects the deformation in the original log-polar image. An image simulator in discrete space remaps a compensated log-polar image by this model. Chapter III proposes Unreliable Feature Omission (UFO), that is, a method for reducing local high frequency noise from the space-variant image using Discrete Wavelet Transform. UFO is simulated and discussed by adding different 3 kinds of white Gaussian noise to the original log-polar image.

\section{ECCENTRICITY COMPENSATOR}

\section{A. Acquisition of Wide-Angle Distorted Foveated Image}

A distorted foveated image $D F$ is computed from a Cartesian target image $I$. The image $D F$ simulates an input image by the fovea sensor. In the following definitions, it is assumed that the image $I$ is on the object plane and is shifted with eccentricity $\theta_{\varepsilon}$ in the $\phi_{\varepsilon}$-direction. The $\theta_{\varepsilon}$ denotes angle between the optical axis of the fovea sensor and the straight line from the optical center $O_{c}$ to the image center $O$ of the image $I$, as in Fig. 2. The image center $O$ is the origin of continuous coordinates $(x, y)$. Discrete coordinates, $\left(x_{d}, y_{d}\right)$, of the image $I$ are computed using an element size $\left(\delta_{x}, \delta_{y}\right)$.

$$
\left\{\begin{array}{l}
x_{d}=\left\lfloor x / \delta_{x}+0.5\right\rfloor \\
y_{d}=\left\lfloor y / \delta_{y}+0.5\right\rfloor
\end{array} .\right.
$$

A direction $(\theta, \phi)$, from a point $P(x, y)$ to the optical center $O_{c}$ of the fovea sensor, is expresses as

$$
\left\{\begin{array}{l}
\theta=\tan ^{-1}\left\{\sqrt{\left(x+\varepsilon \cos \phi_{\varepsilon}\right)^{2}+\left(y+\varepsilon \sin \phi_{\varepsilon}\right)^{2}} / L\right\}, \\
\phi=\tan ^{-1}\left\{\left(y+\varepsilon \sin \phi_{\varepsilon}\right) /\left(x+\varepsilon \cos \phi_{\varepsilon}\right)\right\}
\end{array},\right.
$$

where $L$ is a length from the optical center $O_{c}$ to the object plane, and $\varepsilon$ is positional eccentricity on the object plane.

$$
\varepsilon=L \tan \theta_{\varepsilon},
$$

Continuous coordinates $\left(x^{\prime}, y^{\prime}\right)$ of the distorted foveated image $D F$ are expressed as

$$
\left\{\begin{array}{l}
x^{\prime}=\alpha_{1}\left(r(\theta) \cos \phi-r\left(\theta_{\varepsilon}\right) \cos \left(\phi_{\varepsilon}\right)\right) \\
y^{\prime}=\alpha_{1}\left(r(\theta) \sin \phi-r\left(\theta_{\varepsilon}\right) \sin \left(\phi_{\varepsilon}\right)\right)
\end{array},\right.
$$

where $r(\theta)$ shows the image height of the fovea sensor versus incident angle $\theta, \alpha_{1}$ is magnification of the image, $\theta_{\varepsilon}$ and $\phi_{\varepsilon}$ denote the direction from the image center $O$ to the optical center $O_{c}$. The origin $O^{\prime}$ of the coordinates $\left(x^{\prime}, y^{\prime}\right)$ corresponds to the direction $\left(\theta_{\varepsilon}, \phi_{\varepsilon}\right)$ (that is, the image center $O)$. Discrete coordinates, $\left(x_{d}^{\prime}, y_{d}\right)$, of the image $D F$ are computed using an element size $\left(\delta_{x}^{\prime}, \delta_{y}^{\prime}\right)$.

$$
\left\{\begin{array}{l}
x_{d}^{\prime}=\left\lfloor x^{\prime} / \delta_{x}^{\prime}+0.5\right\rfloor \\
y_{d}^{\prime}=\left\lfloor y^{\prime} / \delta_{y}^{\prime}+0.5\right\rfloor
\end{array} .\right.
$$

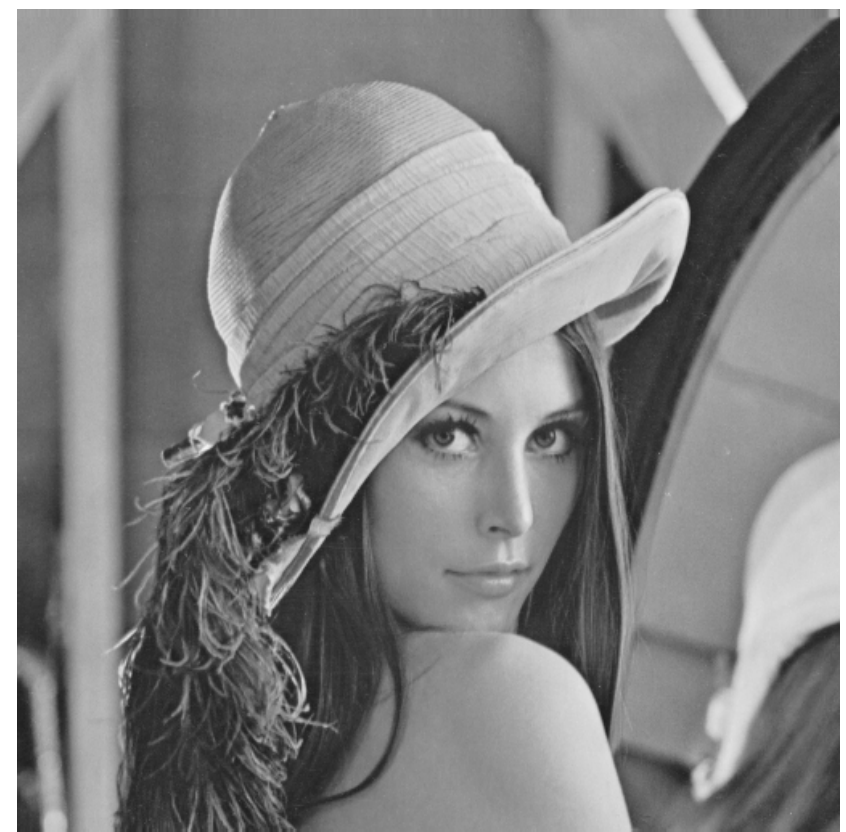

(a) Target image $I$ (of 512x512)
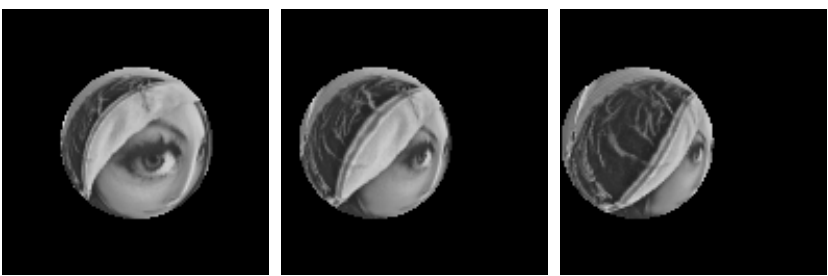

(b) $D F($ of $128 \times 128)$
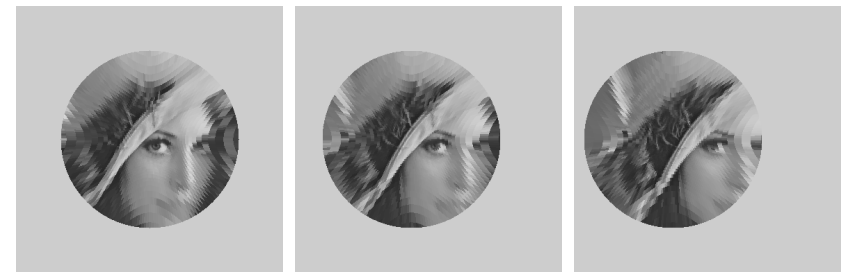

(c) $U D F$
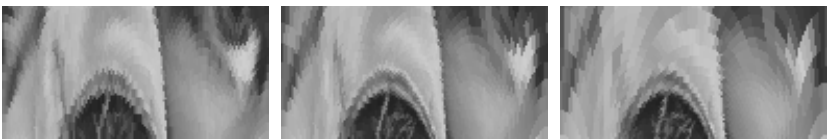

(d) $C L P($ of $128 \times 64)$
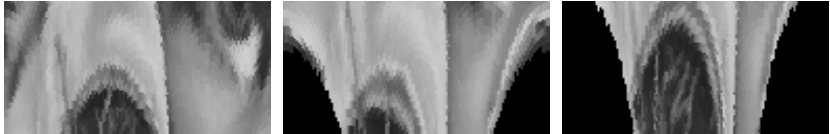

(e) $P_{1}$ (of $\left.128 \times 64\right)$
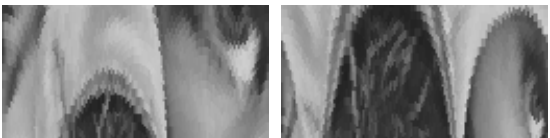

(f) $P_{2}$ (of 128x64)

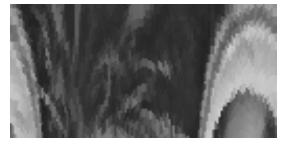

Fig. 1 (a) Target image $I$, (b) distorted foveated image $D F$, (c) undistorted foveated image $U D F$, (d) compensated log-polar image $C L P$, and (e) a polar image $P_{1}$ and (f)the original log-polar image $P_{2}$ in each eccentricity $\theta_{\varepsilon}=0\left[^{\circ}\right.$, $18.69\left[^{\circ}\right]$, and $34.08\left[^{\circ}\right.$ ] from the left 


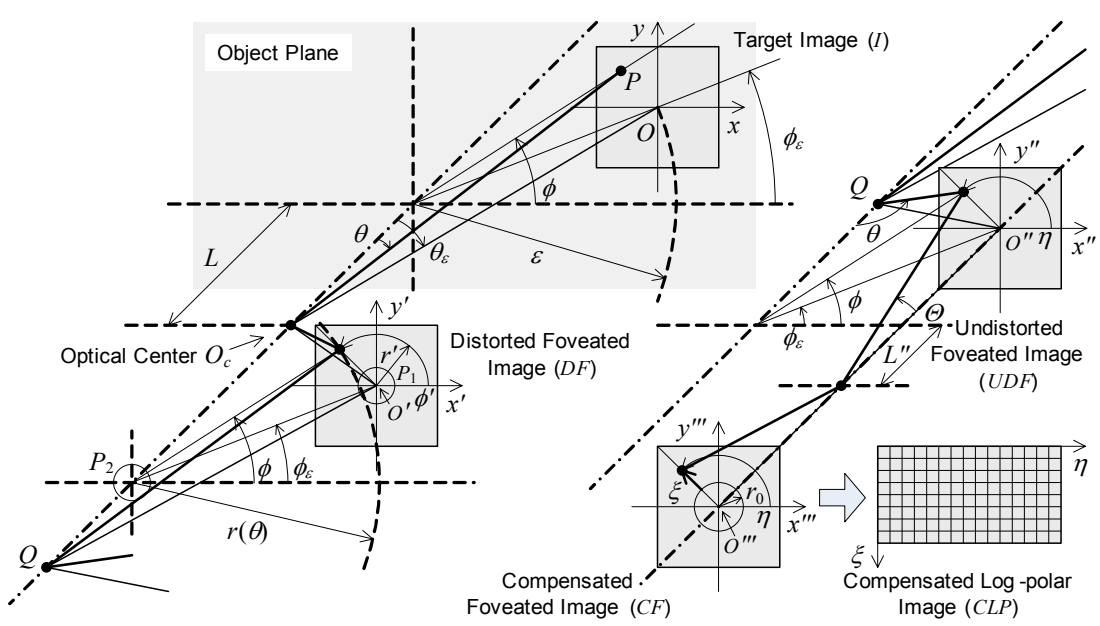

Fig. 2 Coordinate systems (2 points $Q$ are identical with each other)

Advanced Wide Angle Foveated (AdWAF) model [10] is used as a model of the image $D F$ in this paper. This model uses both Cartesian coordinates and logarithmic coordinates in both planar projection and spherical projection. The field of view (FOV) is divided into 4 areas, that is, fovea $\left(0 \leq \theta \leq \theta_{0}\right)$, para-fovea $\left(\theta_{0} \leq \theta \leq \theta_{1}\right)$, near-periphery $\left(\theta_{1} \leq \theta \leq \theta_{2}\right)$, and periphery $\left(\theta_{2} \leq \theta \leq \theta_{\max }\right)$. Figure 3 compares a simulated AdWAF image and a Cartesian image by pinhole camera (PHC) lens model. In this figure, the boundaries of FOV, that is, $\theta_{0}, \theta_{1}$ and $\theta_{2}$, are $9.826\left[^{\circ}\right]$, $19.107\left[^{\circ}\right]$, and $34.715\left[^{\circ}\right]$, respectively. Intensity is changed in order to see each boundary intuitively. The image height $r(\theta)$ of the AdWAF model is defined in the following.

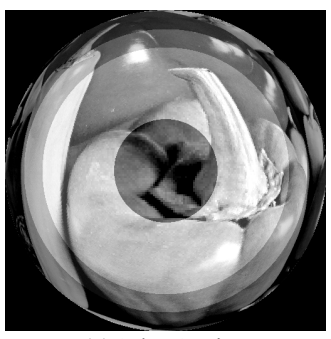

(a)AdWAF image

Fig. 3 Comparison of AdWAF image and Cartesian image
AdWAF model:

$$
r=r_{\max } c_{0} f_{1} \tan \theta \quad\left(0 \leq \theta \leq \theta_{0}\right),
$$

where $f_{1}$ is a focal length for planar projection.

$$
r=r_{\max }\left\{c_{1} \log _{a} f_{1} \tan \theta+d_{1}\right\} \quad\left(\theta_{0} \leq \theta \leq \theta_{1}\right),
$$

where a basis $a$ is defined as $a=\exp \left(1 / f_{1} \tan \theta_{0}\right)$. This corresponds to the brightest area in Fig. 3.

$$
r=r_{\text {max }}\left\{c_{2} \log _{b}\left(f_{2} \theta\right)+d_{2}\right\} \quad\left(\theta_{1} \leq \theta \leq \theta_{2}\right),
$$

where $f_{2}$ is a focal length for spherical projection, and a basis $b$ is defined as $b=\exp \left(1 / f_{2} \theta_{2}\right)$.

$$
r=r_{\max }\left\{c_{3} f_{2} \theta+d_{3}\right\} \quad\left(\theta_{2} \leq \theta \leq \theta_{\max }\right),
$$

where $r_{\max }$ is the maximum image height when $\theta=\theta_{\max }, c_{i}$ $(i=0,1,2,3)$ is a scale modification factor for adjusting the image height, and $d_{i}(i=1,2,3)$ is calculated by continuity of the image height and its magnification.
PHC lens:

$$
\begin{array}{ll}
r & =\frac{r_{\max } c_{0}}{\tan \theta_{\max }} \tan \theta \quad\left(0 \leq \theta \leq \theta_{\max }\right) . \\
L & =\frac{r_{\max }}{\tan \theta_{\max }} .
\end{array}
$$

\section{B. Geometric Modeling of Compensated Log-polar Image}

As shown in Fig. 1(b), the image $D F$ is distorted highly. Continuous coordinates, $\left(x^{\prime \prime}, y^{\prime \prime}\right)$, of the undistorted foveated image $U D F$ are expressed as

$$
\left\{\begin{array}{l}
x^{\prime \prime}=\alpha_{2}\left(L \tan \theta \cos \phi-\varepsilon \cos \phi_{\varepsilon}\right) \\
y^{\prime \prime}=\alpha_{2}\left(L \tan \theta \sin \phi-\varepsilon \sin \phi_{\varepsilon}\right)
\end{array}\right.
$$

where $\alpha_{2}$ is magnification. The origin $O^{\prime \prime}$ of the coordinates $\left(x^{\prime \prime}, y^{\prime \prime}\right)$ corresponds to the image center $O$. Discrete coordinates, $\left(x^{\prime \prime}{ }_{d}, y^{\prime \prime}{ }_{d}\right)$, of the image $U D F$ are computed using an element size $\left(\delta^{\prime \prime}{ }_{x}, \delta^{\prime \prime}{ }_{y}\right)$.

$$
\left\{\begin{array}{l}
x_{d}^{\prime \prime}=\left\lfloor x^{\prime \prime} / \delta_{x}^{\prime \prime}+0.5\right\rfloor \\
y_{d}^{\prime \prime}=\left\lfloor y^{\prime \prime} / \delta_{y}^{\prime \prime}+0.5\right]
\end{array} .\right.
$$

Continuous coordinates, $(\eta, \xi)$, of the compensated log-polar image $C L P$ (that is, a remapped log-polar image after correcting the deformation caused by the eccentricity) are computed from the coordinates $\left(x^{\prime \prime}, y^{\prime \prime}\right)$.

$$
\left\{\begin{array}{l}
\eta=\tan ^{-1}\left(y^{\prime \prime} / x^{\prime \prime}\right) \\
\xi=\alpha_{3}\left(r(\Theta)-r_{0}\right)
\end{array} \quad\left(\theta_{0} \leq \Theta \leq \theta_{1}\right),\right.
$$

where $r_{0}$ is a radius from which the log-polar image starts, $\alpha_{3}$ is magnification, and $\Theta$ is defined as

$$
\Theta=\tan ^{-1}\left(\frac{\sqrt{x^{\prime 2}+y^{\prime \prime 2}}}{L^{\prime \prime}}\right),
$$

where $L^{\prime \prime}=\alpha_{2} L$. Discrete coordinates, $\left(\eta_{d}, \xi_{d}\right)$, of the image $C L P$ are computed using an element size $\left(\delta_{\eta}, \delta_{\xi}\right)$.

$$
\left\{\begin{array}{l}
\eta_{d}=\left\lfloor\eta / \delta_{\eta}+0.5\right. \\
\xi_{d}=\left\lfloor\xi / \delta_{\xi}+0.5\right.
\end{array}\right] \quad\left(\theta_{0} \leq \Theta \leq \theta_{1}\right),
$$

where the maximum integer of $\eta_{d}$ is replaced with 0 (that is, the discrete value $\eta_{d}$ corresponds to $0 \leq \eta<2 \pi$ ).

Cartesian coordinates, $\left(x^{\prime \prime \prime}, y^{\prime \prime \prime}\right)$, of a compensated foveated image $C F$ are expressed as 


$$
\left\{\begin{array}{l}
x^{\prime \prime \prime}=\alpha_{4} r(\Theta) \cos \eta \\
y^{\prime \prime \prime}=\alpha_{4} r(\Theta) \sin \eta
\end{array},\right.
$$

where $\alpha_{4}$ is magnification. Discrete coordinates, $\left(x_{d}^{\prime \prime \prime}, y_{d}^{\prime \prime \prime}\right)$, of the image $C F$ are computed using an element size $\left(\delta^{\prime \prime \prime}{ }_{x}, \delta^{\prime \prime \prime}{ }_{y}\right)$.

$$
\left\{\begin{array}{l}
x_{d}^{\prime \prime \prime}=\left\lfloor x^{\prime \prime \prime} / \delta_{x}^{\prime \prime \prime}+0.5\right\rfloor \\
y_{d}^{\prime \prime \prime}=\left\lfloor y^{\prime \prime \prime} / \delta_{y}^{\prime \prime \prime}+0.5\right.
\end{array}\right] .
$$

\section{Image Simulator in Discrete Space}

In discrete space, image simulator of the proposed compensator outputs the image $C L P$ using the following 2 mappings $f$ and $f_{C L P}$.

$$
\begin{aligned}
f \quad: I \rightarrow D F, & , \text { if } N=0 \\
D F\left(x_{d}^{\prime}, y_{d}^{\prime}\right)=\left\{\begin{array}{cl}
0 & \text {,if } N \geq 1
\end{array},\right. & \frac{1}{N} \sum_{i \in S} I\left(x_{d i}, y_{d i}\right)
\end{aligned}
$$

where $N$ is the number of points $\left(x_{d i}, y_{d i}\right)\left\{i \in S \mid x_{d}^{\prime} \leq x_{d i}^{\prime} \leq x_{d}^{\prime}+1\right.$ $\left.\cap y_{d}^{\prime} \leq y_{d i}^{\prime} \leq y_{d}^{\prime}+1\right\}$ corresponding to each point $\left(x_{d}^{\prime}, y_{d}^{\prime}\right)$. Note that the $N$ is not constant with respect to each point $\left(x_{d}^{\prime}, y_{d}^{\prime}\right)$.

$$
\begin{array}{r}
f_{C L P}: D F \rightarrow C L P, \\
C L P\left(\eta_{d}, \xi_{d}\right)=\left\{\begin{array}{cc}
0 & \text {, if } N=0 \\
\frac{1}{N} \sum_{i \in S_{C L P}} D F\left(x_{d i}^{\prime}, y_{d i}^{\prime}\right), & \text { if } N \geq 1
\end{array},\right.
\end{array}
$$

where $N$ is the number of the points $\left(x_{d i}^{\prime}, y_{d i}^{\prime}\right)\left\{i \in S_{C L P} \mid \eta_{d} \leq \eta_{d i} \leq\right.$ $\left.\eta_{d}+1 \cap \xi_{d} \leq \xi_{d i} \leq \xi_{d}+1\right\}$ corresponding to each point $\left(\eta_{d}, \xi_{d}\right)$.

Some methods for foveation and cortical mapping are known [2]-[4]. The mappings $f$ and $f_{C L P}$ denote foveation and a kind of cortical mapping, respectively. The latter can be called log-polar remapping. These mappings formulize how the $N$-multiple points correspond to each point in the remapped image in addition to geometric modeling. It is noted that the proposed image simulator computes intensity of each point in a different way from the above methods [2]-[4], taking the fovea lens into consideration. This mapping way is discussed again in next chapter, with respect to digitized errors of the remapped image.

\section{Performance Test of Geometry}

In the following simulation, a boundary between fovea and para-fovea is defined as $r_{0}=r_{\max } c_{1} f_{1} \tan \theta_{0}$ in the AdWAF model. Figure 1(a) shows a target image $I$ of $512 \times 512$ [pixels], and Fig. 1(b) shows the distorted foveated image $D F$ of $128 \times 128$ [pixels], simulated from the image $I$ using the mapping $f$ by eq. (7) where the $r_{\max }$ of the AdWAF model is 36 [pixels], in 3 cases of eccentricity $\theta_{\varepsilon}=0\left[^{\circ}\right], 18.69\left[^{\circ}\right]$ and $34.08\left[^{\circ}\right]$ (in condition of $\delta=\delta_{x}=\delta_{y}$ and $\phi_{\varepsilon}=0$ ). Figure 1(d) shows the compensated log-polar image CLP of $128 \times 64$ [pixels] that corresponds to the para-fovea by eq. (6) where $\theta_{0}=9.826\left[^{\circ}\right]$ and $\theta_{1}=19.107\left[^{\circ}\right]$. In order to test the compensator, a polar image $P_{1}$ (Fig. $1(\mathrm{e})$ ) and the original log-polar image $P_{2}$ (Fig. 1(f)) of 128x64 [pixels] are computed from the image $D F$ and compared with the image $C L P$. The image $P_{1}$ has polar coordinates $\left(r^{\prime}, \phi^{\prime}\right)$ around the origin $O^{\prime}$. The image $P_{2}$ has polar coordinates $(r(\theta), \phi)$ around the optical axis. Their discrete images are represented by $P_{1}\left(r_{d}^{\prime}, \phi_{d}^{\prime}\right)$ and $P_{2}\left(r_{d}, \phi_{d}\right)$, respectively. The compensator corrects the deformation of the log-polar image well in each eccentricity, although the resolution of the image CLP distributes anisotropically. It should be noted that this comparison takes practical use of the fovea sensor by the fovea lens and log-polar chip into account. In order to understand effect of the compensator more intuitively, the undistorted foveated image (UDF) is also shown in Fig. 1(c). Note the right eye of the woman is always located nearly at the origin $O^{\prime \prime}$ (center) in each image $U D F$.

The image $C L P$ is estimated using root mean square error (RMSE) from a model log-polar image $L P$, acquired from the image $I$ (Fig.4). The RMSE $E$ is defined as

$$
E=\sqrt{\frac{\|C L P-L P\|^{2}}{N_{\eta} \cdot N_{\xi}}},
$$

where $N_{\eta}$ and $N_{\xi}$ shows the size of the log-polar image.

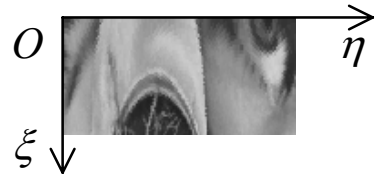

Fig. 4 A model log-polar image $L P$ and its coordinates

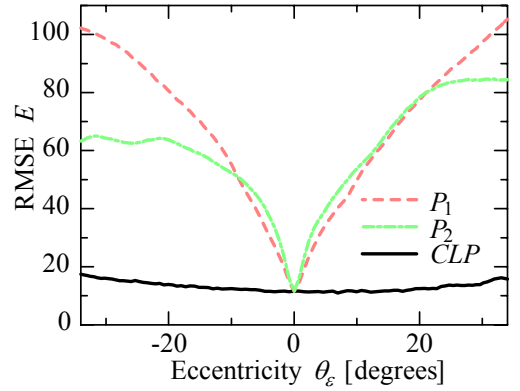

Fig. 5 RMSE of $P_{1}, P_{2}$ and $C L P$ versus $\theta_{\varepsilon}$

Figure 5 compares RMSE of the image $P_{1}$ (broken line), the image $P_{2}$ (dashed line) and the image CLP (solid line) versus the eccentricity $\theta_{\varepsilon}$ (when $\phi_{\varepsilon}=0$ ). The RMSE of the image $C L P$ is smaller in all incident angles than those of the images $P_{1}$ and $P_{2}$. This means that the compensator corrects the deformation in the images $P_{1}$ and $P_{2}$ well. However, the RMSE of the image CLP increases gradually as the eccentricity gets larger, because the intensity of more points in the image $C L P$ is computed from lower-resolution part (like the periphery) of the image $D F$ (the intensity from the lower-resolution part has larger error than higher-resolution one). The RMSE of the image $C L P$ changes by up to $2.54[\%]$, in condition of the eccentricity within $\theta_{\varepsilon}=34.08\left[^{\circ}\right]$.

The log-polar image acquired from a uniform-resolution image such as the image $I$ is space-variant inherently, however, its resolution changes isotropically. When a low-pass filter is used for such space-variant image in order to reduce noise, not only noise but also fine information is lost from the original image. In addition, if the eccentricity exists, the resolution of the image $C L P$ is not only 
space-variant but also anisotropic. In order to estimate the resolution, this paper uses digitized errors, $\Delta \eta$ and $\Delta \xi$, in the $\eta$ and $\xi$-directions of the image $C L P$. They are computed from digitized errors, $\Delta \theta$ and $\Delta \phi$, in the radial and tangential directions of the image $D F$ (as an input image by the fovea sensor), respectively.

$$
\left\{\begin{array}{l}
\Delta \eta=\left|\frac{\partial \eta}{\partial \theta}\right| \cdot \Delta \theta+\left|\frac{\partial \eta}{\partial \phi}\right| \cdot \Delta \phi \\
\Delta \xi=\left|\frac{\partial \xi}{\partial \theta}\right| \cdot \Delta \theta+\left|\frac{\partial \xi}{\partial \phi}\right| \cdot \Delta \phi
\end{array} .\right.
$$

Fourier-Mellin Transform (FMT) is well known as a method for registering RST-invariant feature [18]-[20]. FMT reduces the influence of the eccentricity by extracting translation-invariant property based on Fourier Transform, while its feature is influenced by the anisotropic space-variant resolution because the feature is computed from Cartesian image (see the image $U D F$ ). On the other hand, the proposed compensator computes feature using log-polar geometry as in the image CLP. A method for acquiring feature, more robust to the eccentricity and noise, from the anisotropic space-variant image is proposed in the next chapter.

\section{UnRELIABLE FEATURE OMISSION}

\section{A. Definition for Space-variant Image}

If the image $C L P$ is up-sampled from the low-resolution part of the image $D F$ not fulfilling the sampling theorem, alias occurs as noise. This noise appears in components more than some frequency. Unreliable Feature Omission (UFO) discards such components using Discrete Wavelet Transform (DWT), because it is suitable for local noise reduction from the space-variant image. UFO is applicable also for the anisotropic space-variant resolution caused by the eccentricity, mentioned in the previous chapter. UFO is defined as follows:

1) Define DWT of the image $C L P$ as $\omega$. Its coefficient is expressed as $\omega_{j k, i}^{c}$, where $j$ is resolution level and $c$ denotes diagonal, horizontal and vertical components as $d$, $h$, and $v$, respectively

2) If points in the image, corresponding to each coefficient $\omega_{j k, i}^{c}$, fulfill conditions $H \wedge \Xi, H$ and $\Xi$ in each case of $c=d, h$ and $v$, respectively, discard the coefficient (set it as zero) as to determine a matrix $v$ of wavelet coefficients.

$$
\begin{aligned}
& H: \Delta \eta\left(\frac{k \cdot N_{\eta}}{2^{j-1}}+m, \frac{2 l \cdot N_{\xi}}{2^{j-1}}+n\right)>\frac{4 \pi M_{f}}{2^{j-1}} \\
& \Xi: \Delta \xi\left(\frac{k \cdot N_{\eta}}{2^{j-1}}+m, \frac{2 l \cdot N_{\xi}}{2^{j-1}}+n\right)>\frac{4 N_{\xi} \cdot M_{f}}{2^{j-1}}
\end{aligned},
$$

where $M_{f}$ is a parameter regulating accuracy of the digitized error in sub-pixel order. The $m$ and $n$ are integer determined by the resolution level $j$.

3) Define an image $U$ by Invert Discrete Wavelet Transform (IWDT) of the $v$.

\section{B. Performance Test of Compensated Log-polar Image}

The image $C L P$ is expressed as $D F+e \mapsto^{f_{C L P}} C L P$ when white Gaussian noise (WGN) $e$ is added to the image $D F$. Figure 6 shows the RMSE of the image before UFO (by a broken line), the image $U$ after UFO (by a solid line) versus the eccentricity $\theta_{\varepsilon}$ (when $\phi_{\varepsilon}=0$ ). The RMSE is computed from the model log-polar image $L P$. This simulation uses Haar wavelet for UFO in conditions of $N_{\eta}=2 N_{\xi}=128$, and $M_{f}$ $=1.0$. Circle, triangle and square show cases when the WGN is 0,5 and $10[\%]$, respectively. The RMSE is computed as an average of 100 times repetition.

In each case of the WGN, the RMSE increases gradually as the eccentricity gets larger. It is noted that the RMSE after UFO increases more gently than that before UFO. This means UFO performs better for removing unreliable DWT coefficients as the image $C L P$ is remapped from the lower-resolution part of the image $D F$. When the WGN is 5 and $10[\%]$, UFO increases quality of the image CLP by reducing local noise (UFO works better in case with more noise), even if the space-variant resolution is not isotropic. However, when the WGN is $0[\%]$, the RMSE after UFO is slightly larger than that before UFO. The high level coefficients, removed by UFO, seem to include not only noise but also a part of the original signal. The following 2 reasons are guessed. One is that the distribution of the errors $\Delta \eta$ and $\Delta \xi$ is not approximated sufficiently to a dyadic pyramid. The other is that actual errors of $\Delta \eta$ and $\Delta \xi$ are smaller than estimated errors by eq. (9), because the image $C L P$ uses a sort of moving average when it is remapped from the image $D F$ as by eq. (8). Figure 7 compares with the RMSE when the regulating parameter $M_{f}=1.5$. The RMSE by $M_{f}=1.5$ is much closer to that before UFO than that by $M_{f}=1.0$. This result is consistent with the above guess.

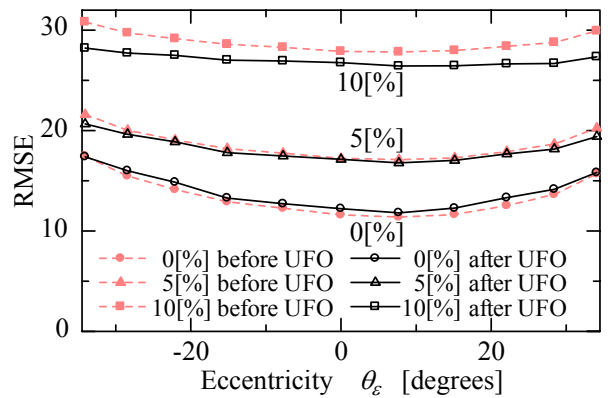

Fig. 6 RMSE before and after UFO

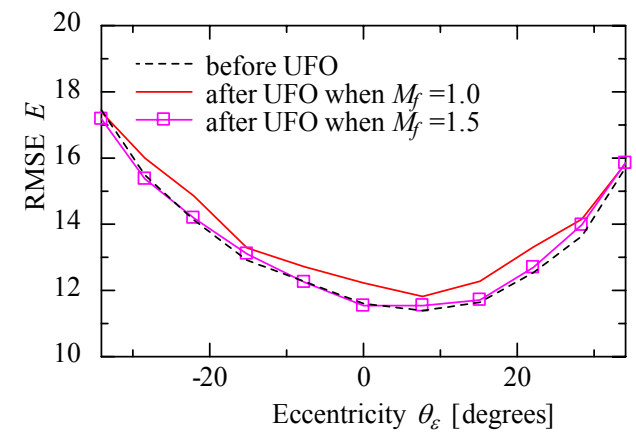

Fig. 7 RMSE when the regulating parameter $M_{F}$ is changed 

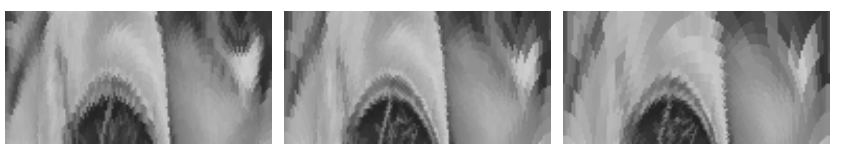

(a) $C L P$ with $0 \% \mathrm{WGN}$ before UFO
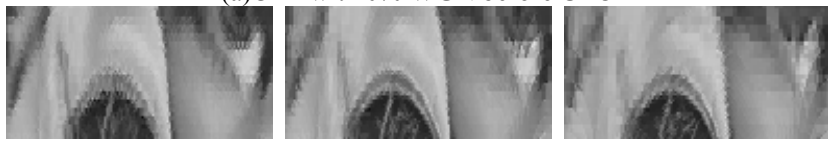

(b)CLP with $0 \% \mathrm{WGN}$ after UFO
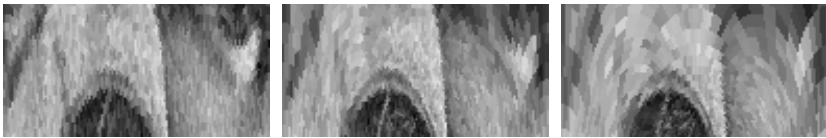

(c) CLP with 5\% WGN before UFO

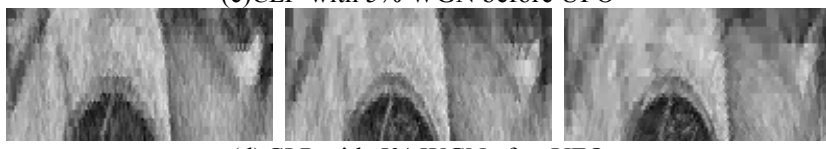

(d)CLP with $5 \% \mathrm{WGN}$ after UFO

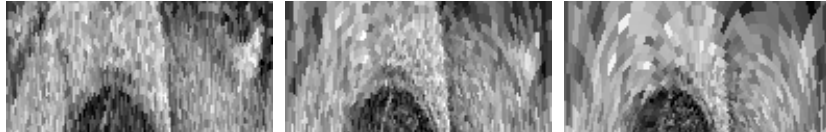

(e)CLP with $10 \% \mathrm{WGN}$ before UFO

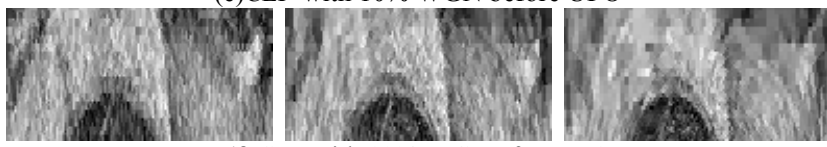

(f) $C L P$ with $10 \% \mathrm{WGN}$ after UFO

Fig. $8 C L P$ before and after UFO

when $\theta_{\varepsilon}=0\left[^{\circ}\right], 18.69\left[^{\circ}\right]$, and $34.08\left[^{\circ}\right]$ from the left
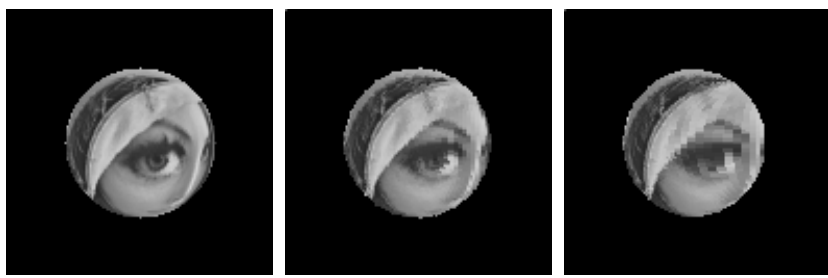

Fig. $9 C F$ when $\theta_{\varepsilon}=0\left[^{\circ}\right], 18.69\left[^{\circ}\right]$, and $34.08\left[^{\circ}\right.$ ] from the left

\section{CONCLUSION}

In the first simulation, it has been demonstrated that the eccentricity compensator performs well for correcting the deformation using log-polar geometry. The RMSE of the image $C L P$ changes by up to $2.54[\%]$, in condition of the eccentricity within $\theta_{\varepsilon}=34.08\left[^{\circ}\right]$. In the second simulation, it has been demonstrated that UFO performs better in case with more white Gaussian noise (WGN), even if the resolution of the image $C L P$ is not isotropic. As future works, each of these methods will be extended to the affine model and different type of wavelet, respectively. Such improvements lead to more practical use and better performance.

Lastly, a mapping $f_{C F}^{\prime}$ from the image $D F$ to the compensated foveated image $C F$ is defined as eq. (10).

$$
\begin{array}{r}
f_{C F}^{\prime}: D F \rightarrow C F, \\
C F\left(x_{d}^{\prime \prime \prime}, y_{d}^{\prime \prime \prime}\right)=\left\{\begin{array}{cc}
0 & , \text { if } N=0 \\
\frac{1}{N} \sum_{i \in S_{C F}^{\prime}} D F\left(x_{d i}^{\prime}, y_{d i}^{\prime}\right), & \text { if } N \geq 1
\end{array},\right.
\end{array},
$$

where $N$ is the number of the points $\left(x_{d i}^{\prime}, y_{d i}^{\prime}\right)\left\{i \in S_{C F}^{\prime} \mid\left(x_{d}^{\prime} \leq x_{d i}^{\prime} \leq\right.\right.$ $\left.\left.x_{d}^{\prime}+1\right) \cap\left(y_{d}^{\prime} \leq y_{d i}^{\prime} \leq y_{d}^{\prime}+1\right)\right\}$ corresponding to each point $\left(x_{d}^{\prime \prime \prime}, y_{d}^{\prime \prime \prime}\right)$.
Figure 9 shows the image $C F$ by eq. (10). The resolution distributes more anisotropically as the eccentricity gets larger. This mapping denotes a combination of the fovea sensor and the eccentricity compensator.

\section{ACKNOWLEDGMENT}

Sota Shimizu sincerely says best regards to Prof. Giulio Sandini and Fabio Berton, the LIRA lab, University of Genova, Italy, Prof. Shinsuke Shimojo and Hao Jiang, California Institute of Technology for their assistance.

\section{REFERENCES}

[1] E.L.Schwartz, "Spatial mapping in the primate sensory projection: Analytic structure and relevance to perception", Biological Cybernetics, Vol.29, pp.181-194, 1977.

[2] G.Sandini and V.Tagliasco, "An anthropomorphic retina-like structure for scene analysis", Computer Graphics and Image Processing, 14, pp.365-372, 1980.

[3] M. Bolduc and M. D. Levine, "A Review of Biologically-Motivated Space-Variant Data Reduction Models for Robotic Vision", Computer Vision and Image Understanding, vol.69, No.2, pp.170-184, 1998.

[4] S. W. Wilson, "On the retino-cortical mapping", International Journal on Man-Machine Studies, vol. 18, pp.361-389, 1983.

[5] F.Berton, G.Sandini and G.Metta, "Anthropomorphic Visual Sensors, Encyclopedia of Sensors", Edited by C.A. Grimes, E.C.Dickey and M.V.Pishko, Vol. 10, pp. 1-16, 2005.

[6] J.Van der Spiegel, G.Kreider, C.Claeys, I.Debusschere, G.Sandini, P.Dario and et al., "A foveated retina-like sensor using CCD technology", Analog VLSI Implementations of Neural Networks. Kluwer, C.Mead and M.Ismail, Boston, 1989.

[7] R.Wodnicki, G.W.Roberts and M.D.Levine, "A foveated image sensor in standard CMOS technology", In Custom Integrated Circuits Conference, Santa Clara, California, May 1995.

[8] S.Shimizu, et.al., "Vision Sensor with Wide angle and High Distortion lens", Video proceedings of IEEE International Conference on Robotics and Automation, Visual Sensing 3, May 1995.

[9] Y.Kuniyoshi, N.Kita, K.Sugimoto, et.al., "A Foveated Wide Angle Lens for Active Vision", Proc. of IEEE International Conference on Robotics and Automation, pp.2982-2988, May 1995.

[10] S,Shimizu, "Wide-Angle Foveation for All-Purpose Use", IEEE transaction on robotics (in review)

[11] A.Bernardino, J. Santos-Victor and G. Sandini, "Foveated active tracking with redundant 2D motion parameters", Robotics and Autonomous System 39, pp. 205-221, 2002.

[12] F. Panerai, C. Capurro and G. Sandini, "Space Variant Vision for an Active Camera Mount", Proc. of SPIE Vol.2488, pp. 284-296, Visual Information Processing IV, June 1995.

[13] C. Capurro, F. Panerai and G. Sandini, "Space Variant Vision for an Active Camera Mount", International Journal of Computer Vision, Vol.24, No.1, pp. 79-94, 1997.

[14] A.Bernardino and J. Santos-Victor, "Binocular Tracking: Integration of Perception and Control", IEEE Transaction on Robotics and Autonomation, Vol. 15, No. 6, pp. 1080-1094, 1999.

[15] S.Mallat, "A theory for muliresolution signal decomposition: the wavelet representation", IEEE Transaction on Pattern Analysis and Machine Intelligence, Vol.11 pp. 674-692, 1989

[16] E.C.Chang and C.Yap, "A wavelet approach to foveating images", Proc. of ACM symposium on Computational Geometry, pp. 397-399, 1997

[17] I. Daubechies, "Ten Lecture on Wavelets", SIAM Philadelphia, 1992

[18] D.Casasent and D.Psaltis, "Position, Rotation and Scale-invariant Optical Correlation", Applied Optics, Vol. 15, pp.1795-1799, 1976.

[19] B.S.Reddy and B.N. Chatterji, "An FFT-based Technique for Translation, Rotation and Scale-Invariant Image Registration", IEEE Transactions on Image Processing, Vol.5, No.8, pp.1266-1271, 1996.

[20] G. Wolberg and S. Zokai, "Robust Image Registration using Log-polar Transform", Proc. of IEEE International Conference on Image Processing, Vol. 1, pp. 493-496, September 2000. 